\title{
Ameliorative role of melatonin against cypermethrin induced hepatotoxicity and impaired antioxidant defense system in Wistar rats
}

\author{
Bhatti GK ${ }^{1}$, Sidhu IPS ${ }^{2}$, Saini NK ${ }^{1}$, Puar $\mathrm{SK}^{3}$, Singh G ${ }^{4}$, Bhatti JS ${ }^{1}$ * \\ ${ }^{I}$ Department of Biotechnology, Sri Guru Gobind Singh College, Sector-26, Chandigarh, India. \\ ${ }^{2}$ Department of Zoology, Sri Guru Gobind Singh College, Sector-26, Chandigarh, India. \\ ${ }^{3}$ Centre for Microbial Biotechnology, Panjab University, Chandigarh, India \\ ${ }^{4}$ Department of Biotechnology, UIET, Panjab University, Chandigarh, India
}

\begin{abstract}
Recently, melatonin has attracted attention because of their strong free radical scavenging and antioxidant properties. The present study was designed to investigate the role of melatonin against cypermethrin induced hepatotoxicity in rats using qualitative, quantitative and biochemical approaches. Adult female Wistar rats were randomly divided into four groups; each group consists of six animals. Control group received vehicle, corn oil; Cypermethrin (CM) [250mg/kg bw]; Melatonin (MEL) [10 mg/kg bw]; CM+MEL treated group. Animals were treated orally, daily for a period of 4 weeks. Animals were sacrificed by decapitation and liver was used for various biochemical and histopathological examinations. The result of this study shows that in vivo administration of cypermethrin caused a significant induction of oxidative damage in liver tissue as evidenced by depletion of proteins, glutathione (GSH) and increased lipid peroxidation in hepatic tissue. Cypermethrin administration significantly enhanced the activities of enzymatic antioxidants (superoxide dismutase (SOD), catalase (CAT), glutathione peroxidase (GPX) glutathione reductase (GR) and glutathione-Stransferase (GST). Cypermethrin intoxication exhibited elevation in the activities of liver marker enzymes such as serum glutamate pyruvate transaminase (SGPT) serum glutamate oxaloacetate transaminase (SGOT) and Lactate dehydrogenase $(L D H)$. Furthermore, the co-administration of melatonin mitigates the hepatotoxicity of cypermethrin by normalizing the biochemical and enzymatic parameters. The biochemical observations were supported by histopathological examination of liver. The findings of this investigation suggest that the melatonin may play a protective role against cypermethrin induced hepatotoxicity and oxidative damage in female albino rats.
\end{abstract}

Keywords: Cypermethrin, Melatonin, Hepatotoxicity, Antioxidant, Oxidative stress.

\section{Introduction}

The widespread use of pesticides worldwide for public health protection and agricultural pest control has resulted in severe environmental pollution and health hazards. Currently, India is the largest producer of pesticides in Asia and ranks twelfth in the world for the use of pesticides. A vast majority of Indian population is engaged in agriculture as cultivators, farm owners and laborers. Pesticide poisoning has become a major reason of morbidity and mortality in developing countries. Recent extrapolations of data from developing countries suggest that pesticide poisoning causes more deaths than infectious diseases [1-3]. People are directly exposed to pesticides through dermal contact and inhalation and indirectly through the food chain. The pyrethroids represents a group of synthetic insecticides constituting $>30 \%$ of insecticide used worldwide $[4,5]$ in agricultural, domestic and veterinary applications [6]. In recent years, they have gained popularity due to their high efficacy against target species, their relatively low mammalian toxicity and rapid biodegradability. Cypermethrin, a type II pyrethroid, is being extensively used for the pest management and animal husbandry practices [7]. Cypermethrin is metabolized in the liver via hydrolytic ester cleavage and oxidative pathways by the cytochrome P-450 enzymes forming cyanohydrins that decompose further to cyanides and aldehydes [8]. These substances induce free radical generation which are responsible for increasing oxidative stress in mammals $[9,10]$. Previous studies have revealed lipid peroxidation as one of the molecular mechanisms involved in pesticide-induced toxicity in rats [11-14]. Cypermethrin intoxication prolongs the opening of voltage-sensitive sodium channels causing hypo-polarization and hyper-excitation of the neurons and induces neurotoxicity [15-17].

Nature produces an array of antioxidants which prevents the production of free radicals and protect against oxidative damage induced by various pesticides. Recently, melatonin ( $N$-acetyl-5-methoxytryptamine), a major secretory product of the pineal gland, has been shown to be an effective antioxidant and free radical scavenger [18-22]. Because of its small size and lipophilicity, melatonin crosses biological membranes easily, thus, reaching all compartments of the cell. Numerous reports have documented protective actions of melatonin 
in various models of oxidative stress due to its high efficacy as a free radical scavenger and indirect antioxidant $[18,23-29]$. Previous studies revealed that melatonin stimulates a host of antioxidative enzymes and protect the cells against oxidative damage induced by pesticide toxicity in rats. To date, there are no available data investigating the antioxidant potency of melatonin against cypermethrin induced toxicity in rats. Thus, present investigation was undertaken to further explore the possible ameliorative role of melatonin against oxidative damage induced by cypermethrin in female Wistar rats.

\section{Material And Methods}

2.1 Chemicals: All the chemicals used in this study were of analytical grade and were purchased from Sigma, St. Louis, USA and Sisco Research Laboratory (SRL), Mumbai, India. Technical-grade cypermethrin was obtained as a gift from Rallis India Ltd.

2.2 Experimental animals and study design: Female Wistar rats, weighing 150-200 grams were procured from Central Animal House of Panjab University, Chandigarh. Animals were housed in plastic cages, fed a standard laboratory diet and water ad libitum, exposed to a $12 \mathrm{~h}$ light/dark cycle. Animals were allowed to acclimatize in the laboratory environment for 10 days before start of treatments. The protocols were approved by Animal Ethics Committee of Panjab University, Chandigarh. The animals were randomly divided into 4 groups, each comprising of six animals and orally administered the respective doses using corn oil as a vehicle. Control group: Animals were given $1.0 \mathrm{ml}$ of corn oil.

Melatonin (MEL) group: Animals were orally supplemented with melatonin (10mg/kg bw).

Cypermethrin (CM) treated group: Animals were treated with cypermethrin $(25 \mathrm{mg} / \mathrm{kg} \mathrm{bw})$.

$C M+M E L$ treated group: Animals were given cypermethrin $(25 \mathrm{mg} / \mathrm{kg}$ body weight) along with melatonin $(10 \mathrm{mg} / \mathrm{kg}$ bw).

After 4 weeks of treatment, animals were sacrificed by decapitation under ether anesthesia following overnight fast. Blood and liver tissues collected were then used for various biochemical assays and histopathological examinations. The liver tissue was washed twice with ice cold $0.1 \mathrm{M}$ phosphate buffered saline (1:9), $\mathrm{pH}$ 7.4, blotted, dried and weighed. A small portion of the tissue was used for histopathological examinations. The remaining tissue immediately was stored at $-20^{\circ} \mathrm{C}$ for further studies. A $10 \%$ tissue homogenate was prepared in $50 \mathrm{mM}$ Tris-HCl solution ( $\mathrm{pH}$ 7.4) by homogenizing the tissue using Potter-Elvehjem glass homogenizer. The homogenate was centrifuged at $6000 \mathrm{~g}$ for $15 \mathrm{~min}$ at $4^{\circ} \mathrm{C}$ to remove the cell debris and the post mitochondrial supernatant (PMS) obtained was then used for enzymatic assays.

\subsection{Biochemical Measurements}

2.3.1 Estimation of Proteins: The protein content in liver homogenate were quantified by the method of Lowry et al. [30], using bovine serum albumin (BSA) as a standard.

3.2.2 Phospholipids Content: Phospholipids were estimated by the method of Barlett as modified by Marinetti [31]. The organic phosphorus is converted to inorganic phosphorus by digesting with perchloric acid. When this acid hydrolysate is treated with molybedate, it forms phosphomolybidic acid with inorganic phosphorus. The phosphomolybedate formed is reduced by the addition of 1,2,4-aminonapthol sulphonic acid(ANSA) to give a blue color, whose intensity is proportional to the amount of inorganic phosphorus.

2.3.3 Cholesterol Content: Cholesterol was estimated in the liver homogenate according to the method of Zlatkis et al. [32]. The reaction involves initial dehydration of cholesterol to 3,5-cholestadiene which react with $\mathrm{FeCl}_{3}-\mathrm{H}_{2} \mathrm{SO}_{4}$ to form a colored complex, intensity of which was measured colorimetrically at $540 \mathrm{~nm}$.

2.3.4 Total lipids: Total lipids were determined by the method of Fringes and Dunn [33] in which color was developed by phosphovanillin in the presence of conc. $\mathrm{H}_{2} \mathrm{SO}_{4}$ and the absorbance was measured at $540 \mathrm{~nm}$.

2.3.5 Measurement of lipid peroxidation: As an index of lipid peroxidation, MDA level was determined in the liver homogenate by the method of Draper and Hadley [34]. This procedure was based on the formation of a pink chromophore, at $532 \mathrm{~nm}$, following the reaction of thiobarbituric acid (TBA) with degradation products of peroxidized lipids, releasing malondialdehyde (MDA). TBA-MDA chromophore has been taken as an index of lipid peroxidation. Lipid peroxidation was calculated using molar extinction coefficient of MDA $\left(1.56 \times 10^{5} \mathrm{M}^{-1}\right.$ $\left.\mathrm{cm}^{-1}\right)$ at $532 \mathrm{~nm}$.

2.3.6 Measurement of reduced glutathione (GSH) content: GSH content were quantified in liver homogenate using 5, 5'-dithiobis-2-nitrobenzoicacid (DNTB) as the complexing agent [35]. This method is based on reduction of DNTB by free -SH groups to form a yellow colored complex, 2-nitro-5-mercaptobenzoic 
acid. The glutathione contents were expressed as nmoles of $\mathrm{GSH} / \mathrm{mg}$ protein using the molar extinction coefficient of GSH $\left(13.6 \times 10^{6} \mathrm{M}^{-1} \mathrm{~cm}^{-1}\right)$ at $412 \mathrm{~nm}$.

\subsection{Enzymatic Assay}

2.4.1 Liver marker enzymes: Serum glutamate pyruvate transaminase (SGPT), serum glutamate oxaloacetate transaminase (SGOT), alkaline phosphatase (ALP) and lactate dehydrogenase (LDH) enzymes were assayed in the serum using a commercially available spectrophotometric enzymatic kit and analyzed on semi-autoanalyzer.

2.4.2 Catalase (CAT) activity: CAT activity was spectrophotometrically measured by the rate of decomposition of $\mathrm{H}_{2} \mathrm{O}_{2}$ by Catalase [36]. CAT activity was expressed as $\mu$ moles of $\mathrm{H}_{2} \mathrm{O}_{2}$ decomposed $/ \mathrm{min} / \mathrm{mg}$ protein.

2.4.3 Superoxide Dismutase (SOD) activity: SOD activity was assayed by a method based on generation of superoxide anions by the oxidation of hydroxylamine hydrochloride [37]. The reduction of nitro blue tetrazolium (NBT) to blue formazon by superoxide anions was measured at $560 \mathrm{~nm}$ under aerobic conditions. SOD activity was expressed as $\mathrm{U} / \mathrm{mg}$ protein.

2.4.4 Glutathione-S-transferase (GST) activity: GST activity was assayed by a standard procedure [38] using 1-chloro-2,4-dinitrobenzene (CDNB) as a substrate which absorb maximum at $340 \mathrm{~nm}$ and have an extinction coefficient of $9.6 \mathrm{mM}^{-1} \mathrm{~cm}^{-1}$.

2.4.5 Glutathione peroxidase (GPx) activity: GPx activity was measured by the modified method of Paglia and Valentine [39]. GPx catalyzes the oxidation of glutathione by cumene hydroperoxide. In the presence of glutathione reductase and NADPH, the oxidized glutathione is immediately converted to the reduced form with

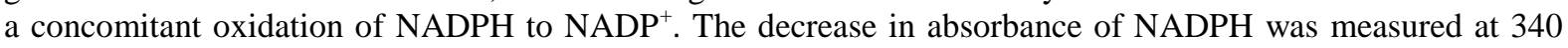
nm wavelength. GPx activity was expressed as nmol NADPH oxidized/min/mg protein.

2.4.6 Glutathione reductase (GR) activity: GR activity was determined by following the oxidation of NADPH to NADP during the reduction of oxidized glutathione [40]. GR activity was expressed as nmol of $\mathrm{NADPH}$ oxidized/min/mg protein.

2.5 Histopathology of Liver: The liver tissues were isolated from all the experimental rats and were gently rinsed with physiological saline solution $(0.9 \% \mathrm{NaCl})$ to remove blood and debris adhering to the tissue. The liver samples were fixed in $10 \%$ buffered formalin, processed through graded alcohols and xylene and embedded in paraffin blocks. Tissue sections were cut at $5 \mu \mathrm{m}$ at multiple levels and stained with haematoxylin and counter stained with eosin (dissolved in 95\% alcohol). After dehydration and clearing, DPX mounted slides were examined and observed under photomicroscope.

2.6 Statistical Analysis: The data are expressed as Mean \pm Standard Deviation (SD). Differences among experimental groups were calculated by using one-way analysis of variance (ANOVA). In all experiments, p $<0.05$ was considered statistically significant. The data were analyzed by using SPSS for Windows version 16.

\section{Results}

3.1 Evaluation of body and organ weight: During the 4 weeks of treatment, none of the animals in any experimental group died. Also the animals were observed daily and gross visual observations of rats of CM treated rats showed reduced physical activity as compared to control, MEL and CM+MEL treated groups.

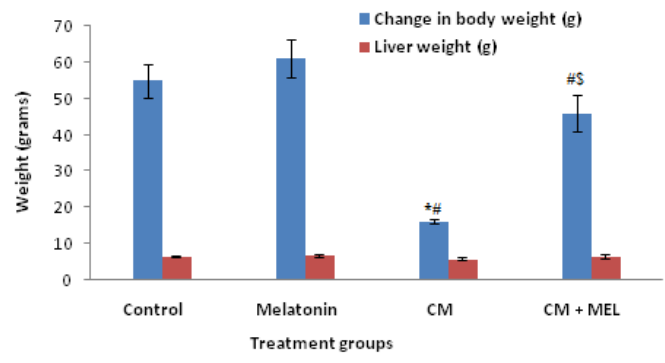

and CM+MEL treated groups. Significant loss in the body weight of CM treated rats was observed when

Figure 1: Effect of in vivo administration of cypermethrin on body weight and liver weight of experimental rats.

Values are expressed as Mean $\pm S D$. "Significantly different from control group $(P<0.05)$. ${ }^{\#}$ Significantly different from melatonin treated group $(P<0.05)$. ${ }^{\$}$ Significantly different from cypermethrin treated group $(p<0.05)$.

There was a significant decrease in body weight of animals treated with cypermethrin alone as compared to control, MEL 
compared with the Control groups (Fig. 1). No significant change was observed in the liver weight of rats treated with cypermethrin. However a significant decrease in the relative liver weight of animals treated with cypermethrin was observed as compared to control group $(\mathrm{p}<0.05)$.

3.2 Effect of cypermethrin toxicity on biochemical parameters: The effect of in vivo administration of cypermethrin and melatonin on biochemical parameters are given in the Table 1. Cypermethrin exposure caused a significant decline in protein content and elevation in phospholipids, cholesterol and total lipids, when compared with the control group. Although melatonin supplementation significantly raised the amount of proteins in CM+MEL treated group. But no significant change was observed in lipids levels when compared with control group

Table 1: Effect of in vivo administration of cypermethrin on biochemical parameters in experimental animals.

\begin{tabular}{|l|l|l|l|l|}
\hline Treatment Groups & Total Proteins $(\mathrm{mg} / \mathrm{ml})$ & Phospholipids $(\mathrm{mg} / \mathrm{ml})$ & Cholesterol $(\mathrm{mg} / \mathrm{dl})$ & Total Lipids $(\mathrm{mg} / \mathrm{g}$ tissue) \\
\hline Control & $8.61 \pm 0.36$ & $7.47 \pm 0.28$ & $27.81 \pm 2.22$ & $115.69 \pm 10.62$ \\
\hline Melatonin (MEL) treated & $8.93 \pm 0.45$ & $7.53 \pm 0.46$ & $44.12 \pm 3.02$ & $132.29 \pm 5.12$ \\
\hline Cypermethrin (CM) treated & $5.59 \pm 0.73^{* \#}$ & $12.45 \pm 0.66^{* \#}$ & $36.20 \pm 2.93^{* \#}$ & $174.87 \pm 8.32^{* \#}$ \\
\hline CM+MEL treated & $7.20 \pm 0.39^{\$}$ & $7.68 \pm 0.33^{\$}$ & $28.40 \pm 2.31^{\$}$ & $112.07 \pm 7.03^{\# \$}$ \\
\hline
\end{tabular}

Values are expressed as Mean $\pm S D$. "Significantly different from control group $(P<0.05)$. ${ }^{\#}$ Significantly different from melatonin treated group $(P<0.05)$. ${ }^{\$}$ Significantly different from cypermethrin treated group $(p<0.05)$.

3.3 Lipid Peroxidation: The effect of in vivo administration of cypermethrin on lipid peroxidation in liver is shown in Fig. 2. Exposure of animals with cypermethrin resulted in a significant increase in MDA level compared to control animals $(\mathrm{p}<0.05)$.

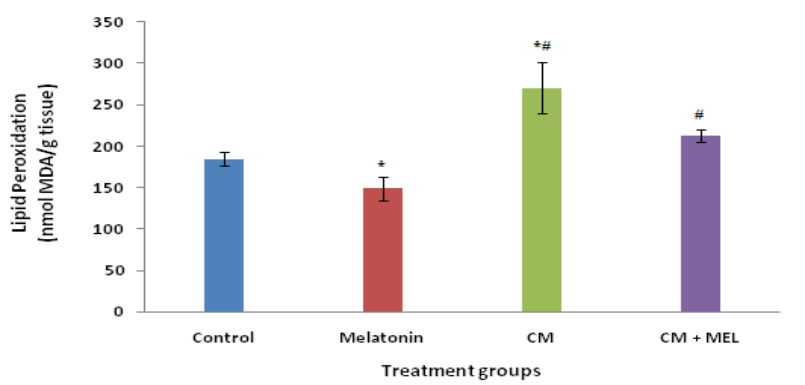

Fig. 2: Effect of in vivo administration of cypermethrin on lipid peroxidation in experimental rats. Values are expressed as Mean $\pm S D$. "Significantly different from control group (P<0.05). " Significantly different from melatonin treated group $(P<0.05)$. ${ }^{\$}$ Significantly different from cypermethrin treated group $(p<0.05)$.

A significant decrease in the LPO was observed when animals were supplemented with melatonin along with cypermethrin, compared to cypermethrin treated group. The treatment with melatonin was more effective in terms of reducing lipid peroxidation and the oxidative damage induced by cypermethrin.

3.4 Glutathione Content: The effect of in vivo administration of cypermethrin on glutathione level in liver homogenate of experimental groups is given in Fig. 3. Comparing with the control group, a significant depletion in GSH level was observed in cypermethrin treated rats $(\mathrm{p}<0.05)$. However, melatonin supplementation along with cypermethrin significantly raised the GSH level in CM+MEL group rats when compared with CM treated rats.

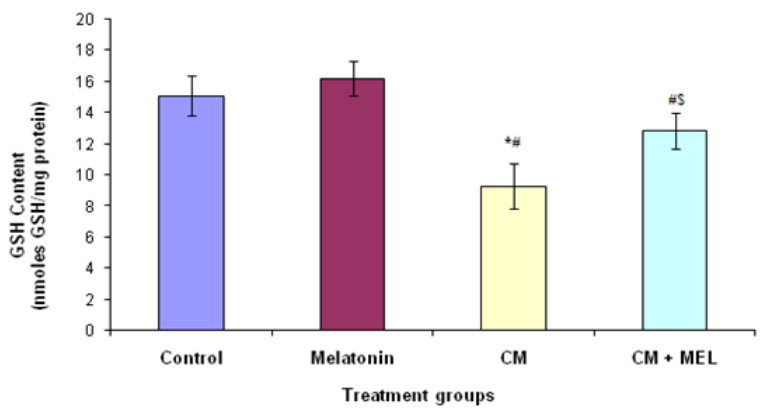

Fig. 3: Cypermethrin induced changes in GSH level of experimental rats.

Values are expressed as Mean $\pm S D$. "Significantly different from control group $(P<0.05)$. " Significantly different from melatonin treated group $(P<0.05)$. ${ }^{\$}$ Significantly different from cypermethrin treated group $(p<0.05)$.

These results indicate that cypermethrin intoxication lower the GSH level and administration of melatonin along with cypermethrin showed recovery in their GSH content as compared to cypermethrin treated group.

3.5 Liver Marker Enzymes: The effect of melatonin supplementation on liver marker enzymes in the serum level of liver marker enzymes in cypermethrin treated female Wister rats is shown in Table 2. Cypermethrin intoxication resulted in a significant increase in the levels of SGOT and SGPT, which were 
reduced to near normal values in the CM+MEL treated group, when compared with control group. In contrast to this, there was a significant decrease in the ALP activity in cypermethrin treated rats as compared to controls.

Table 2: Effect of in vivo administration of melatonin on liver marker enzymes of cypermethrin intoxicated female Wistar rats.

\begin{tabular}{|c|c|c|c|c|}
\hline Groups & SGPT (U/L) & SGOT (U/L) & ALP (U/L) & LDH (U/L) \\
\hline Control & $14.90 \pm 0.43$ & $13.40 \pm 0.74$ & $27.50 \pm 1.43$ & $855.83 \pm 18.14$ \\
\hline Melatonin & $14.10 \pm 1.66$ & $14.90 \pm 1.32$ & $32.40 \pm 2.19$ & $847.84 \pm 9.21$ \\
\hline CM & $16.10 \pm 1.14$ & $18.90 \pm 2.27^{* \#}$ & $21.40 \pm 2.42^{* \#}$ & $861.65 \pm 32.30$ \\
\hline CM+MEL & $15.70 \pm 1.33$ & $15.80 \pm 1.80^{\$}$ & $26.40 \pm 0.92^{\# \$}$ & $852.43 \pm 24.86$ \\
\hline
\end{tabular}

Values are expressed as Mean $\pm S D$. "Significantly different from control group $(P<0.05)$. ${ }^{\#}$ Significantly different from melatonin treated group $(P<0.05)$. ${ }^{\$}$ Significantly different from cypermethrin treated group $(p<0.05)$.

Furthermore, cypermethrin exposure resulted a significant increase in LDH level as compared to control. No significant difference was observed in control and MEL groups. However, supplementation of melatonin to CM intoxicated rats showed decrease in LDH level when compared with CM treated rats.

3.6 Antioxidant Defense Enzymes: Fig. 4 (a-b) shows the effect of in vivo administration of melatonin on antioxidant enzymes in all the experimental rats. There was significant elevation in the activities of GST in CM treated group as compared to control or melatonin treated rats. A similar trend was observed in the activities of antioxidant enzymes, CAT, SOD and GR. However, significant recovery in antioxidant enzymes was observed in CM+MEL treated rats. Opposite to this, a significant decrease in GPx activity was observed in cypermethrin treated rats as compared to control group and but melatonin supplementation in CM+MEL treated group shows normal values for GPx.

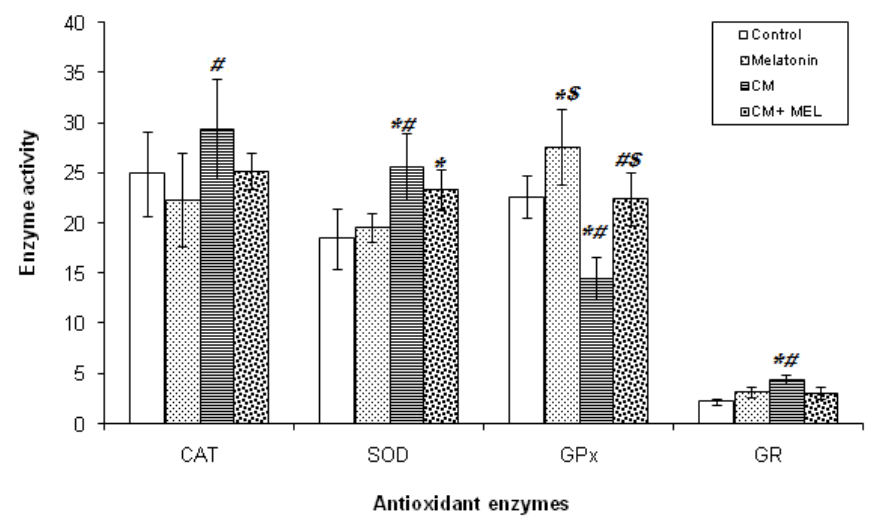

Fig. 4 (a): Effect of in vivo administration of cypermethrin on antioxidant defense enzymes.

Values are expressed as Mean $\pm S D$. "Significantly different from control group $(P<0.05)$. "Significantly different from melatonin treated group $(P<0.05)$. ${ }^{\$}$ Significantly different from cypermethrin treated group ( $p<0.05)$.

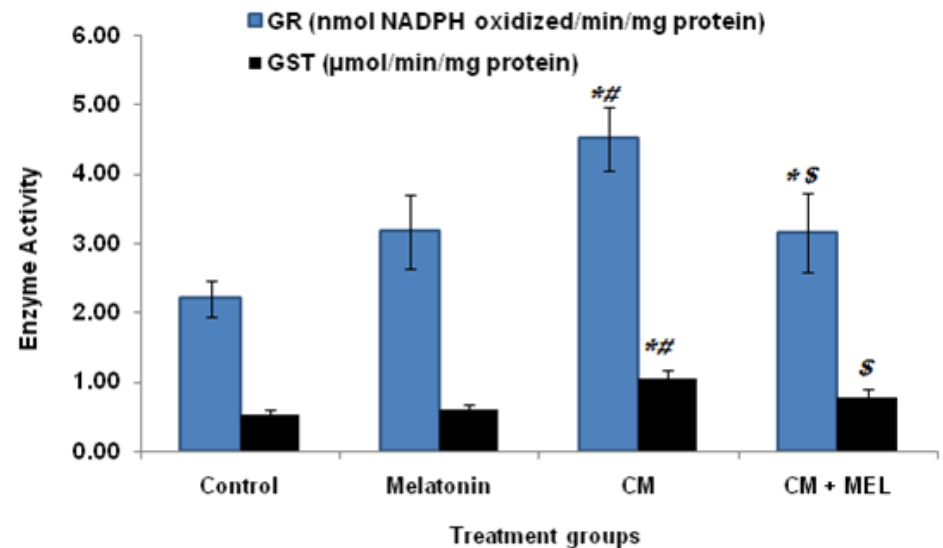

Fig. 4 (b): Effect of in vivo administration of cypermethrin on Glutathione Reductase and Glutathione-S-Transferase enzyme. Values are expressed as Mean \pm SD. *Significantly different from control group $(P<0.05) .{ }^{\#}$ Significantly different from melatonin treated group $(P<0.05)$. ${ }^{\$}$ Significantly different from cypermethrin treated group ( $p<0.05)$.

3.7 Histoarchitecture Examinations of Liver: Fig. 5 (A-D) shows the effect of in vivo administration of cypermethrin on histopathology of liver in experimental rats. Marked hepatocytes degeneration and presence of hepatocytes in intralobular vein, cytoplasmic and nuclear vacuolization, necrosis and karyorrhexis was observed in the liver of $\mathrm{CM}$ exposed rats as compared to the normal histopathological examination of liver tissue of 
control as well as melatonin treated groups. However, histopathology of liver of CM+MEL treated animals showed significant recovery of the damage induced by cypermethrin when compared with CM treated rats.

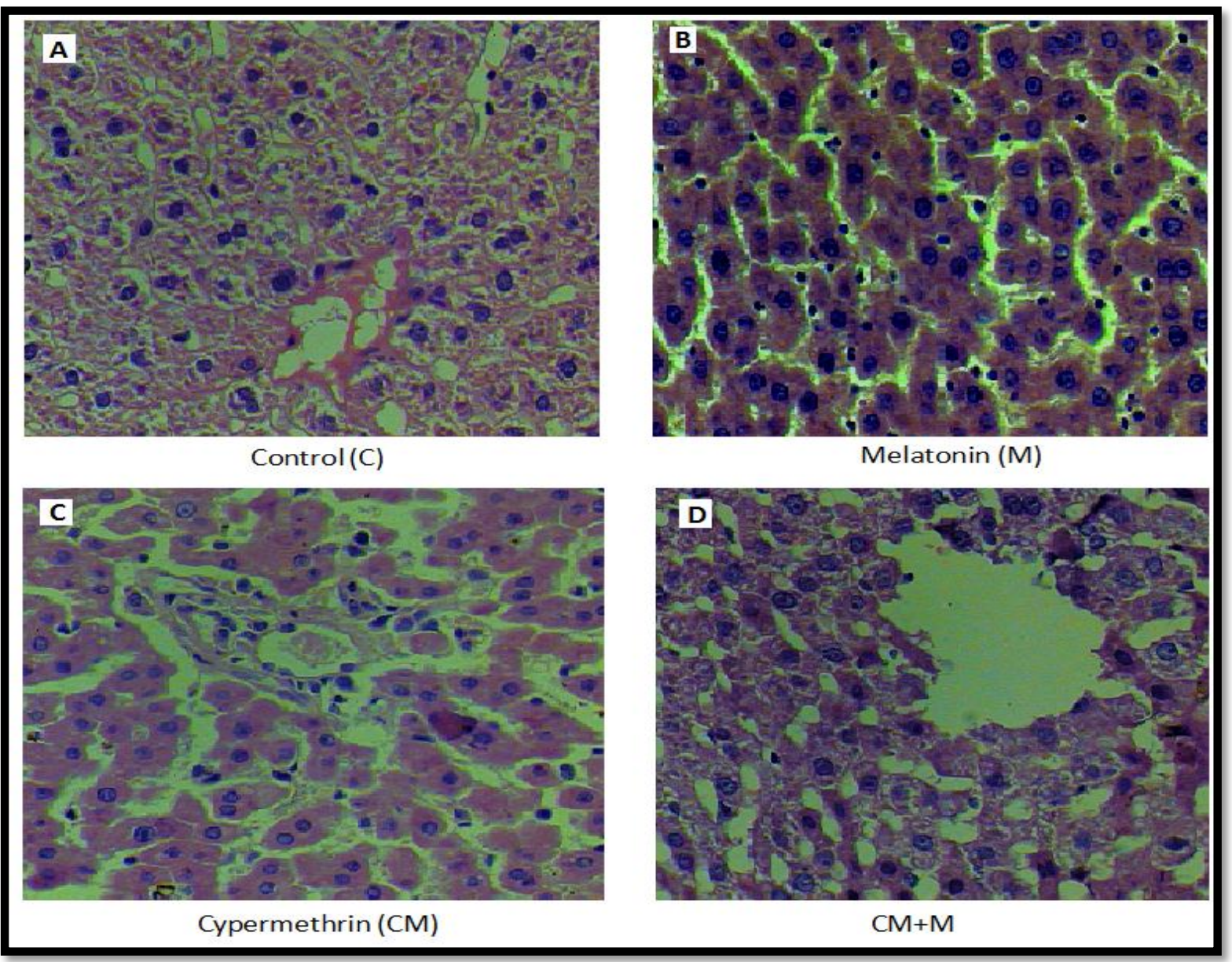

Figure 5: Histoarchitecture of liver tissue (40X)

\section{Discussion}

Free radicals are fundamental to any biochemical process and are continuously produced in the body. Exposure to pesticides caused generation of reactive oxygen species (ROS) which induces oxidative stress and alterations in antioxidant defense mechanisms [14]. The cells have many ways to alleviate the deleterious effects of oxidative stress, either by repairing the damage or by directly diminishing the occurrence of oxidative damage by means of enzymatic and non-enzymatic antioxidants. These include vitamins, dietary flavonoids, melatonin, $\alpha$-lipoic acid etc. However, amounts of these protective devices present under normal physiological conditions are solely sufficient only to cope with the normal threshold of physiological rate of free-radical generation. Therefore, any additional burden of free radicals, either from an indigenous or exogenous source leads to the oxidative imbalance leading to oxidative stress [41]. So the present study was planned to investigate the action of melatonin against cypermethrin induced hepatotoxicity in rats. The significant differences in the body weight of cypermethrin treated rats and control reflects the deleterious effects of cypermethrin. Similar observations were reported in previous studies [14, 42-44]. However, concomitant use of melatonin attenuated the body and liver weight on cypermethrin intoxicated rats. This suggests that melatonin plays a protective role against cypermethrin induced toxicity in rats.

Liver plays a major role in the detoxification of xenobiotics, protein synthesis, and regulation of cell functioning. Disturbed liver homeostasis under oxidative imbalance is sufficient to alter the normal physiological functioning of any organism [45]. Our findings suggest that cypermethrin intoxication strongly disrupt normal hepatic function in rats. Decrease in total protein observed in cypermethrin intoxicated rats may be associated with the decrease in the number of hepatocytes, which in turn may result in the decreased hepatic capacity to synthesize proteins and consequently decreased liver weight as observed in this study. 
The impaired activities of liver marker enzymes such as SGOT, SGPT and ALP are directly implicated in the extent of hepatic damage induced by toxic compounds. The elevated levels of SGOT, SGPT and LDH enzymes in cypermethrin intoxicated rats, as observed in the present study, may be attributed to the disturbed structural integrity of the liver. SGOT and SGPT are sensitive inductors of hepatocellular damage under oxidative stress caused by xenobiotics compounds [46]. The reduced hepatic ALP may be a consequence of cell membrane damage induced by cypermethrin intoxication. Elevation in LDH activity indicates cell lysis and death.

The perturbed lipid composition i.e. total lipids, cholesterol and phospholipids in cypermethrin treated rats may be due to the increased lipogenesis, which reflects abnormal carbohydrate metabolism. Lipid peroxidation and altered lipid composition are known to disturb structural integrity of the membrane that might in turn affect the activity of enzymes of metabolic processes in hepatic tissues. The protein depletion in cypermethrin intoxicated rats may be due to the lysis of structural proteins as evident from the histopathology of liver [46]. Recovery of protein content in melatonin supplemented rats may be due to the strong antioxidant properties of melatonin which completely normalize the liver functions in the experimental groups. Melatonin has been shown to be an efficient protector of DNA, proteins and lipids in cellular membrane [47, 48].

The toxic manifestations induced by various pesticides may be associated with enhanced production of free radicals. Measuring the extent of lipid peroxidation indirectly reflects the degree to which cell membranes are damaged by free radicals. The increase in extent of lipid peroxidation in the present study could possibly be due to the generation of free radicals following cypermethrin exposure. However, co-administration of melatonin resulted in a significant reduction in lipid peroxidation in hepatic tissues of cypermethrin treated rats. This may be due to the potency of melatonin to scavenge the free radicals which are generated during the oxidation of unsaturated lipids and lead to the propagation of lipid peroxidation [49-51]. Recent investigations reported that cypermethrin induces lipid peroxidation and oxidative stress in hepatic tissue [44, 52-54]. This suggests that increased lipid production may be one of the molecular mechanisms involved in the cypermethrin induced hepatotoxicity in rats.

Several pharmacological strategies have been used to reduce the production of reactive oxygen species with the intent of lowering pesticide toxicity. Glutathione is considered to be an important cellular antioxidant which has various biological functions, one of which is scavenging of ROS, free radicals, and reactive metabolites [55]. It possess highly reactive sulfhydryl (-SH) group, which like other thiols, acts nonenzymatically as a free radical acceptor to counteract oxidative damage. The decline in the levels of glutathione in the present study might be due to increased utilization of GSH for conjugation and or participation of GSH as an antioxidant in terminating the free radicals produced by cypermethrin induced toxicity. Various OPIs have been reported to decrease the GSH level both in vivo and in vitro in rats $[14,56]$. It has been reported that GSH play an important role in protecting cells from xenobiotics induced tissue injury [55, 57]. Simultaneous administration of melatonin resulted in marked protection against cypermethrin induced hepatotoxicity as evidenced by the reversal of lipid peroxidation and glutathione depletion. This may be due to the higher efficacy of melatonin in scavenging various free radicals [58].

Enzymatic antioxidants such as SOD, CAT, GST, GPx and GR have been shown to scavenge free radicals and ROS. In the current study, Increase in the activities of these antioxidant enzymes in the hepatic tissue of cypermethrin intoxicated rats was probably a response towards increased ROS generation. Furthermore, it has been reported that the mRNA levels for the genes encoding antioxidant proteins, such as GPx and SOD are also up regulated significantly in cypermethrin treated mice [52]. Similarly, generation of free radicals following cypermethrin treatment up-regulated the CAT activity, as observed in the present study. Coadministration of melatonin markedly attenuated oxidative stress induced by cypermethrin and maintains the SOD, CAT, GST, GR and GPx activities in hepatic tissues, indicating controlled generation of $\mathrm{H}_{2} \mathrm{O}_{2}$ or its scavenging directly by melatonin, as observed in a previous study [59]. This effect might result in reduced accumulation of lipid and protein oxidation products and hence ameliorate oxidative stress [21]. The activity of antioxidant enzymes, maintained within controlled level by co-administration of melatonin, indicating the potent role of melatonin as an antioxidant [60]. These actions of melatonin may be related to its ability to limit oxidative stress and DNA damage [61]. These observations assert the hypothesis that the production of oxidative damage is one of the principal mechanisms involved in cypermethrin toxicity and melatonin play a pivotal role in the prevention of oxidative damage induced by cypermethrin. Recent studies revealed the protective role of melatonin against the oxidative damage induced by radiations $[60,62]$ and other xenobiotics compounds $[63,64]$.

Present study results revealed that subchronic administration of cypermethrin resulted in histopathological alterations in rat liver. These changes may be attributed to increased peroxidation of polyunsaturated fatty acids. Previous studies have suggested that cypermethrin induces histopathological changes in liver [46, 65]. These changes are possibly due to oxidative stress induced by cypermethrin toxicity. However, co-administration of melatonin resulted in normalization of liver tissue. 


\section{Conclusion:}

The present investigations demonstrate that in vivo administration of cypermethrin induces oxidative stress that results in the induction of LPO, depletion of GSH content alteration in activities of liver marker enzymes and antioxidant defence enzymes, suggesting that ROS may be involved in the toxic effects of cypermethrin. The changes in the cholesterol and phospholipids contents may be responsible for higher lipid peroxidation. However, supplementation of melatonin restored the biochemical and morphological alterations in hepatic tissue induced by cypermethrin intoxication. Thus, present investigation suggests that melatonin is a potent antioxidant against cypermethrin induced toxicity in rats.

\section{Acknowledgement:}

Authors are very thankful to University Grants Commission, Govt. of India for financial support provided under Major Research Project. We are very thankful to Panjab University, Chandigarh for providing animal house facilities.

\section{References}

[1] D. Gunnell and M. Eddleston, Suicide by intentional ingestion of pesticides: a continuing tragedy in developing countries, International Journal of Epidemiology, 32(6), 2003, 902-909.

[2] I. Hashmi and A.D. Khan, Adverse health effects of pesticide exposure in agricultural and industrial workers of developing country, in: M. Stoytcheva (Ed.) Pesticides - The Impacts of Pesticides Exposure, InTech 2011, 155-178.

[3] M. Eddleston, L. Karalliedde, N. Buckley, R. Fernando, G. Hutchinson, G. Isbister, F. Konradsen, D. Murray, J.C. Piola, N. Senanayake, R. Sheriff, S. Singh, S.B. Siwach and L. Smit, Pesticide poisoning in the developing world--a minimum pesticides list, Lancet, 360(9340), 2002, 1163-1167.

[4] D.M. Soderlund and J.R. Bloomquist, Neurotoxic actions of pyrethroid insecticides, Annual Review of Entomology, 34, 1989, 7796.

[5] H.P. Vijverberg and J. van den Bercken, Annotation. Action of pyrethroid insecticides on the vertebrate nervous system, Neuropathology and Applied Neurobiology, 8(6), 1982, 421-440.

[6] K. Prasamthi, Muralidhara and P.S. Rajini, Fenvalerate-induced oxidative damage in rat tissues and its attenuation by dietary sesame oil, Food and Chemical Toxicology, 43(2), 2005, 299-306.

[7] R. Jayakumar, A. Nagarjuna, T. Deuraju and R. Jayantha, Alteration of haematological Profiles due to cypermethrin Toxicosis in Rana hexadactyla, Journal of the Indian Society of Toxicology, 4(2), 2008, 973-3558

[8] B. Wielgomas and J. Krechniak, Effect of $\alpha$-cypermethrin and chlorpyrifos in a 28 day study on free radical parameters and cholinesterase activity in Wistar rats, Polish Journal of Environmental Studies, 16, 2007, 91-95.

[9] S. Flodstrom, L. Warngard, S. Ljungquist and U.G. Ahlborg, Inhibition of metabolic cooperation in vitro and enhancement of enzyme altered foci incidence in rat liver by the pyrethroid insecticide fenvalerate, Archives in Toxicology, 61(3), 1988, 218-223.

[10] J. Klimek, Cytochrome P-450 involvement in the NADPH-dependent lipid peroxidation in human placental mitochondria, Biochim Biophys Acta, 1044(1), 1990, 158-164

[11] N. Yilmaz, M. Yilmaz and I. Altuntas, Diazinon-induced brain toxicity and protection by vitamins E plus C, Toxicology and Industrial Health, 28(1), 2012, 51-57.

[12] P. Sankar, A.G. Telang and A. Manimaran, Protective effect of curcumin on cypermethrin-induced oxidative stress in Wistar rats, Experimental Toxicology and Pathology, 64(5), 2012, 487-493.

[13] F.M. El-Demerdash, Lipid peroxidation, oxidative stress and acetylcholinesterase in rat brain exposed to organophosphate and pyrethroid insecticides, Food and Chemical Toxicology, 49(6), 2011, 1346-1352.

[14] G.K. Bhatti, R. Kiran and R. Sandhir, Modulation of ethion-induced hepatotoxicity and oxidative stress by vitamin E supplementation in male Wistar rats, Pesticide Biochemistry and Physiology, 98, 2010, 26-32.

[15] J.T. Eells and M.L. Dubocovich, Pyrethroid insecticides evoke neurotransmitter release from rabbit striatal slices, The Journal of Pharmacology and Experimental Therapeutics, 246(2), 1988, 514-521.

[16] T. Narahashi, J.M. Frey, K.S. Ginsburg and M.L. Roy, Sodium and GABA-activated channels as the targets of pyrethroids and cyclodienes, Toxicology Letters, 64-65 Spec No. 1992, 429-436.

[17] M.L. Kirby, K. Castagnoli and J.R. Bloomquist, In vivo effects of deltamethrin on dopamine neurochemistry and the role of augmented neurotransmitter release, Pesticide Biochemistry and Physiology, 65, 1999, 160-168.

[18] R.J. Reiter, Oxidative damage in the central nervous system: protection by melatonin, Progress in Neurobiology, 56(3), 1998, 359384.

[19] R. Reiter, L. Tang, J.J. Garcia and A. Munoz-Hoyos, Pharmacological actions of melatonin in oxygen radical pathophysiology, Life Sciences, 60(25), 1997, 2255-2271.

[20] D.S. Maharaj, B.D. Glass and S. Daya, Melatonin: new places in therapy, Bioscience Reports, 27(6), 2007, 299-320.

[21] J.S. Bhatti, I.P. Sidhu and G.K. Bhatti, Ameliorative action of melatonin on oxidative damage induced by atrazine toxicity in rat erythrocytes, Molecular and Cellular Biochemistry, 353(1-2), 2011, 139-149.

[22] R.J. Reiter, D.X. Tan, A.-C. D., S. Burkhardt and M. Karbownik, Melatonin: mechanisms and actions as an antioxidant, Current Topics in Biophysics, 24(2000, 171-183.

[23] J. Xu, S. Sun, W. Wei, J. Fu, W. Qi, L.C. Manchester, D.X. Tan and R.J. Reiter, Melatonin reduces mortality and oxidatively mediated hepatic and renal damage due to diquat treatment, Journal of Pineal Research, 42(2), 2007, 166-171.

[24] H. Wang, W. Wei, S.Y. Zhang, Y.X. Shen, L. Yue, N.P. Wang and S.Y. Xu, Melatonin-selenium nanoparticles inhibit oxidative stress and protect against hepatic injury induced by Bacillus Calmette-Guerin/lipopolysaccharide in mice, Journal of Pineal Research, 39(2), 2005, 156-163.

[25] A. Wakatsuki, Y. Okatani, K. Shinohara, N. Ikenoue, C. Kaneda and T. Fukaya, Melatonin protects fetal rat brain against oxidative mitochondrial damage, Journal of Pineal Research, 30(1), 2001, 22-28.

[26] I. Tunez, C. Munoz Mdel, M. Feijoo, J.R. Munoz-Castaneda, I. Bujalance, M.E. Valdelvira and P. Montilla Lopez, Protective melatonin effect on oxidative stress induced by okadaic acid into rat brain, Journal of Pineal Research, 34(4), 2003, 265-268.

[27] M. Sun, P.P. Xu, Y. Ren, Y.F. Li, Y.F. Zhong and H. Yan, [Protective effect of melatonin on oxidative damage by deltamethrin in rat brain], Zhonghua Lao Dong Wei Sheng Zhi Ye Bing Za Zhi, 25(3), 2007, 155-158. 
[28] G. Sener, A.O. Sehirli and G. Ayanoglu-Dulger, Melatonin protects against mercury(II)-induced oxidative tissue damage in rats, Pharmacology and Toxicology, 93(6), 2003, 290-296.

[29] F.G. Princ, A.G. Maxit, C. Cardalda, A. Batlle and A.A. Juknat, In vivo protection by melatonin against delta-aminolevulinic acidinduced oxidative damage and its antioxidant effect on the activity of haem enzymes, Journal of Pineal Research, 24(1), 1998, 1-8.

[30] O.H. Lowry, N.J. Rosebrough, A.L. Farr and R.J. Randall, Protein measurement with the Folin phenol reagent, Journal of Biological Chemistry, 193(1), 1951, 265-275.

[31] G.R. Bartlett, Phosphorus assay in column chromatography, Journal of Biological Chemistry, 234(3), 1959, 466-468.

[32] A. Zlatkis, B. Zak and A.J. Boyle, A new method for the direct determination of serum cholesterol, Journal of Laboratory and Clinical Medicine, 41(3), 1953, 486-492.

[33] C.S. Frings and R.T. Dunn, A colorimetric method for determination of total serum lipids based on the sulfo-phospho-vanillin reaction, American Journal of Clinical Pathology, 53(1), 1970, 89-91.

[34] H.H. Draper and M. Hadley, Malondialdehyde determination as index of lipid peroxidation, Methods in Enzymology, 186(1990, 421-431.

[35] G.L. Ellman, Tissue sulfhydryl groups, Archives of Biochemistry and Biophysics, 82(1), 1959, 70-77.

[36] H. Luck, Catalase, in: H.U. Bergmeyer (Ed.) Methods of enzymatic analysis Academic Press, New York, $1971,885-893$.

[37] Y. Kono, Generation of superoxide radical during autoxidation of hydroxylamine and an assay for superoxide dismutase, Archives of Biochemistry and Biophysics, 186(1), 1978, 189-195.

[38] W.H. Habig, M.J. Pabst and W.B. Jakoby, Glutathione S-transferases. The first enzymatic step in mercapturic acid formation, Journal of Biological Chemistry, 249(22), 1974, 7130-7139.

[39] D.E. Paglia and W.N. Valentine, Studies on the quantitative and qualitative characterization of erythrocyte glutathione peroxidase, Journal of Laboratory and Clinical Mededicine, 70(1), 1967, 158-169.

[40] D.M. Goldberg and R.J. Spooner, Glutathione reductase, in: H.U. Bergmeyer (Ed.) Methods Enzymol, Verlag Chemie, Basel, Switzerland, 1983, 258-265.

[41] A.K. Tiwari, Antioxidants: New-generation therapeutic base for treatment of polygenic disorders, Current Science, 86(8), 2004, 1092-1102.

[42] T.M. Heikal, H.Z. Ghanem and M.S. Soliman, Protective effect of green tea extracts against dimethoate induced DNA damage and oxidant/antioxidant status in male rats, Biohealth Science Bulletin, 3(2011, 1-11.

[43] S. Dahamna, A. Belguet, D. Bouamra, A. Guendouz, M. Mergham and D. Harzallah, Evaluation of the toxicity of cypermethrin pesticide on organs weight loss and some biochemical and histological parameters, Communications in agricultural and applied biological sciences, 76(4), 2011, 915-921.

[44] G.K. Sangha, K. Kaur, K.S. Khera and B. Singh, Toxicological effects of cypermethrin on female albino rats., Toxicology International, 18(1), 2011, 5-8.

[45] R.H. Hinton and P. Grasso, Hepatotoxicity., in: B. Ballantyne, T. Marrs, T. Syversen (Eds.) General and applied toxicology, Groves Dictionaries Inc., New York (NY) 2000, 853-892.

[46] B. Bhushan, P.N. Saxena and N. Saxena, Biochemical and histological changes in rat liver caused by cypermethrin and betacyfluthrin, Archives of Industrial Hygiene and Toxicology, 64(1), 2013, 57-67.

[47] S. Lopez-Burillo, D.X. Tan, J.C. Mayo, R.M. Sainz, L.C. Manchester and R.J. Reiter, Melatonin, xanthurenic acid, resveratrol, EGCG, vitamin C and alpha-lipoic acid differentially reduce oxidative DNA damage induced by Fenton reagents: a study of their individual and synergistic actions, Journal of Pineal Research, 34(4), 2003, 269-277.

[48] M. Karbownik, D.X. Tan and R.J. Reiter, Melatonin reduces the oxidation of nuclear DNA and membrane lipids induced by the carcinogen delta-aminolevulinic acid, International Journal of Cancer, 88(1), 2000, 7-11.

[49] D.X. Tan, L.D. Chen, B. Poeggler, L.C. Manchester and R.J. Reiter, Melatonin: a potent, endogenous hydroxyl radical scavenger., Endocrinology Journal, 1, 1993, 57-60.

[50] E. Gilad, S. Cuzzocrea, B. Zingarelli, A.L. Salzman and C. Szabo, Melatonin is a scavenger of peroxynitrite, Life Science, 60(10), 1997, PL169-174.

[51] C. Pieri, M. Marra, F. Moroni, R. Recchioni and F. Marcheselli, Melatonin: a peroxyl radical scavenger more effective than vitamin E, Life Science, 55(15), 1994, PL271-276.

[52] Y. Jin, S. Zheng, Y. Pu, L. Shu, L. Sun, W. Liu and Z. Fu, Cypermethrin has the potential to induce hepatic oxidative stress, DNA damage and apoptosis in adult zebrafish (Danio rerio), Chemosphere, 82(3), 2011, 398-404.

[53] V. Muthuviveganandavel, P. Muthuraman, S. Muthu and K. Srikumar, A study on low dose cypermethrin induced histopathology, lipid peroxidation and marker enzyme changes in male rat., Pesticide Biochemistry and Physiology, 91(1), 2008, 12-16.

[54] B. Giray, A. Gurbay and F. Hincal, Cypermethrin-induced oxidative stress in rat brain and liver is prevented by vitamin E or allopurinol, Toxicol Lett, 118(3), 2001, 139-146.

[55] D.J. Reed and M.W. Fariss, Glutathione depletion and susceptibility, Pharmacological Reviews, 36(2 Suppl), 1984, 25S-33S

[56] A. Hazarika, S.N. Sarkar, S. Hajare, M. Kataria and J.K. Malik, Influence of malathion pretreatment on the toxicity of anilofos in male rats: a biochemical interaction study, Toxicology, 185(1-2), 2003, 1-8.

[57] G. Wu, Y.Z. Fang, S. Yang, J.R. Lupton and N.D. Turner, Glutathione metabolism and its implications for health, Journal of Nutrition, 134(3), 2004, 489-492.

[58] G.H. El-Sokkary, A.A. Nafady and E.H. Shabash, Melatonin administration ameliorates cadmium-induced oxidative stress and morphological changes in the liver of rat, Ecotoxicology and Environmental Safety, 73(3), 2010, 456-463.

[59] P. Venkataraman, K. Selvakumar, G. Krishnamoorthy, S. Muthusami, R. Rameshkumar, S. Prakash and J. Arunakaran, Effect of melatonin on PCB (Aroclor 1254) induced neuronal damage and changes in $\mathrm{Cu} / \mathrm{Zn}$ superoxide dismutase and glutathione peroxidase-4 mRNA expression in cerebral cortex, cerebellum and hippocampus of adult rats, Neuroscience Research, 66(2), 2010, 189-197.

[60] A. Shirazi, E. Mihandoost, G. Ghobadi, M. Mohseni and M. Ghazi-Khansari, Evaluation of radio-protective effect of melatonin on whole body irradiation induced liver tissue damage, Cell Journal, 14(4), 2013, 292-297.

[61] S.G. Ferreira, R.A. Peliciari-Garcia, S.A. Takahashi-Hyodo, A.C. Rodrigues, F.G. Amaral, C.M. Berra, S. Bordin, R. Curi and J. Cipolla-Neto, Effects of melatonin on DNA damage induced by cyclophosphamide in rats, Brazilian Journal of Medical and Biological Research, 46(3), 2013, 278-286.

[62] E. Kucuktulu, Protective effect of melatonin against radiation induced nephrotoxicity in rats, Asian Pacific Journal of Cancer Prevention, 13(8), 2012, 4101-4105.

[63] K.M. Lee, I.C. Lee, S.H. Kim, C. Moon, S.H. Park, D.H. Shin, S.H. Kim, S.C. Park, H.C. Kim and J.C. Kim, Melatonin attenuates doxorubicin-induced testicular toxicity in rats, Andrologia, 44 Suppl 1, 2012, 796-803. 
[64] C. Contini Mdel, N. Millen, M. Gonzalez and S. Mahieu, Melatonin prevents oxidative stress in ovariectomized rats treated with aluminium, Biological Trace Element Research, 144(1-3), 2011, 924-943.

[65] G. Grewal, P.K. Verma, V.J. Dhar and A.K. Srivastav, Toxicity of subacute oral administration of cypermethrin in rats with special reference to histopathological changes, International Journal of Green Pharmacy, 3(4), 2009, 293-299. 